\title{
Model-Based Quantification of Small Tubular Structures in 3D Vascular Images
}

\author{
Stefan Wörz ${ }^{1}$, Hendrik von Tengg-Kobligk ${ }^{2,3}$, Karl Rohr $^{1}$ \\ ${ }^{1}$ Dept. Bioinformatics and Functional Genomics, Biomedical Computer Vision Group, \\ University of Heidelberg, BIOQUANT, IPMB, and DKFZ Heidelberg \\ ${ }^{2}$ Dept. Diagnostic and Interventional Radiology, University of Heidelberg \\ ${ }^{3}$ Dept. Radiology, German Cancer Research Center (DKFZ) Heidelberg \\ s.woerz@dkfz.de
}

\begin{abstract}
We introduce a new 3D tubular intensity model in conjunction with a model fitting scheme for accurate segmentation and quantification of small tubular structures in $3 \mathrm{D}$ vascular images. The tubular model is formulated based on principles of the image formation process. Compared to previous straight models, the new model allows to accurately represent curved tubular structures, to directly estimate the local curvature, as well as to more accurately estimate the parameters of tubular structures. We have successfully applied our approach to 3D synthetic images and 3D CTA vascular images.
\end{abstract}

\section{Introduction}

In modern radiology, different angiographic imaging modalities such as CTA and MRA are used to visualize the human vascular system. Accurate quantification of human vessels is important in different applications (e.g., diagnosis of pathologies). In addition to quantifying the contrast, radius, and centerline of vessels, a number of applications (e.g., analysis of tumor vasculature) require to quantify a vessel's tortuosity, which is related to the (local) curvature of the centerline.

In previous work on $3 \mathrm{D}$ vessel segmentation, different types of approaches have been proposed, for example, approaches based on differential measures (e.g., $[1,2]$ ) and approaches based on deformable models (e.g., [3, 4]). While being computationally efficient, a disadvantage of differential measures is their sensitivity to noise. On the other hand, approaches based on deformable models generally exploit contour information of anatomical structures. Alternatively, deformable models using 3D parametric intensity models have been suggested (e.g., $[5,6])$. Parametric intensity models describe the image intensities of a structure within a region-of-interest (ROI), and are generally defined by an analytic function comprising a certain number of parameters. So far, different types of tubular models have been proposed (e.g., [5, 6]). However, these approaches utilize straight tubular models, i.e., the curvature of vessels is not explicitly modeled. 
In this contribution, we introduce a new curved tubular intensity model in conjunction with a model fitting scheme for accurate segmentation and quantification of small vessels in 3D tomographic images. The model comprises parameters for the local contrast, width, 3D position, and 3D orientation. Moreover, the model includes a parameter for the local (extrinsic) curvature, which describes the local radius of curvature of a tubular structure. In contrast to previous intensity-based approaches, which utilize straight tubular models [5, 6], a curved tubular model has three major advantages. First, using a curved model we can more accurately represent the intensity profile of curved structures, thus the model is more realistic for medical applications. Second, based on our model we can directly estimate the local curvature of tubular structures by model fitting. And third, using a curved model instead of a straight model also improves the accuracy of estimating the remaining model parameters such as the radius and centerline of the tube. In comparison to our approach, in previous work often the local curvature is not computed at all $[5,6]$, or the curvature is computed in a subsequent step based on the estimated centerline.

\section{Methods}

For our 3D intensity model of small tubular structures, we assume a physical image formation process where the intensity structures are generated by blurring of ideal step-shaped structures. For thin structures with sizes below image resolution, the unsmoothed structures can be represented by Dirac delta functions $\delta$. The central idea of our new model is to model the unsmoothed structure by a Dirac ring. By Gaussian smoothing of the Dirac ring we obtain a Gaussian ring. A 3D ROI of the Gaussian ring represents a thin curved tubular structure, where the radius of the ring determines the radius of curvature $R_{\kappa}$. The curvature $\kappa$ is given by $\kappa=R_{\kappa}^{-1}$. More formally, we define a Dirac ring as a series of shifted 3D Dirac delta functions $\delta$ along a circle of radius $R_{\kappa}$ in the $x z$-plane. The center of the ring (center of curvature) is located at $\mathbf{x}_{\kappa}=\left(R_{\kappa}, 0,0\right)$. Using the Gaussian function $G_{\sigma}(x)=(\sqrt{2 \pi} \sigma)^{-1} \exp \left(-x^{2} /\left(2 \sigma^{2}\right)\right)$, the Gaussian ring is obtained by a $3 \mathrm{D}$ convolution

$$
g_{\mathrm{GR}}\left(\mathbf{x}, R_{\kappa}, \sigma\right)=\left(G_{\sigma}(x) \cdot G_{\sigma}(y) \cdot G_{\sigma}(z)\right) * \operatorname{Ring}\left(\mathbf{x}, R_{\kappa}\right)
$$

We have derived an analytic solution of the multiple integral in (1)

$$
g_{\mathrm{GR}}(\mathbf{x})=\frac{\left|R_{\kappa}\right| \sqrt{2 \pi}}{\sigma} e^{\frac{\left(x-R_{\kappa}\right)^{2}+y^{2}+z^{2}+R_{\kappa}^{2}}{-2 \sigma^{2}}} I_{0}\left(\frac{R_{\kappa}}{\sigma^{2}} \sqrt{\left(x-R_{\kappa}\right)^{2}+z^{2}}\right)
$$

where $I_{0}$ is a modified Bessel function of the first kind. Including the intensity levels $a_{0}$ and $a_{1}$ as well as a $3 \mathrm{D}$ rigid transform $\mathcal{R}$ with rotation parameters $\boldsymbol{\alpha}=(\alpha, \beta, \gamma)$ and translation parameters $\mathbf{x}_{0}=\left(x_{0}, y_{0}, z_{0}\right)$, we obtain the final parametric intensity model $g_{M, \mathrm{GR}}(\mathbf{x}, \mathbf{p})=a_{0}+\left(a_{1}-a_{0}\right) g_{\mathrm{GR}}\left(\mathcal{R}\left(\mathbf{x}, \boldsymbol{\alpha}, \mathbf{x}_{0}\right)\right)$ with a total of 10 parameters.

If we analyze (2) in detail, it turns out that the Bessel function $I_{0}$ grows exponentially with increasing argument whereas the exponential function exp 
Fig. 1. Segmentation result for a synthetic 3D spiral (a) shown as $3 \mathrm{D}$ visualization and as 2D sections using (b) a previous straight model and (c) our new curved model, where the centerline (black) and the inner and outer contours (white) are highlighted, as well as (d) the error $e_{\mathbf{x}_{0}}$ (in voxels) of the centerline position along the spiral (in voxels) for the previous straight model (grey) and our new curved model (black).

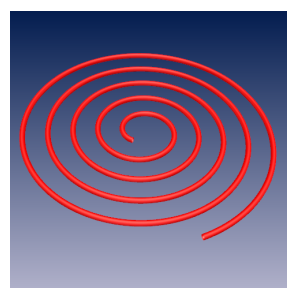

(a)

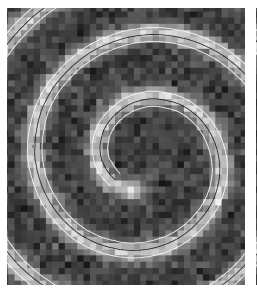

(b)

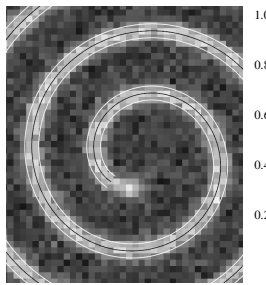

(c)

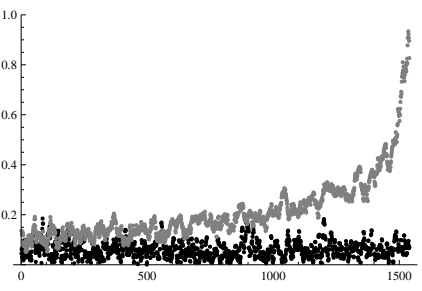

(d)

decays exponentially (approaching zero). In the continuous formula (2), these effects cancel out each other in the product, yielding an overall moderate value. However, numerically computing the values of exp and $I_{0}$ separately can lead to numerical instabilities because of computational over- or underflows. To avoid these over- and underflows we use an approximation of $I_{0}$ for larger arguments, which is based on an exponential function.

To segment a vessel, we apply an incremental approach. For each segment along a vessel, we use a model fitting approach based on least-squares fitting of the $3 \mathrm{D}$ curved tubular model $g_{M}$, GR to the image intensities $g(\mathbf{x})$ within a spherical 3D ROI. For minimization we apply the method of Levenberg-Marquardt. As fitting result we obtain estimates of the model parameters p. Based on the estimated position $\mathbf{x}_{\mathbf{0}}$, orientation $\boldsymbol{\alpha}$, and curvature $\kappa$, we can directly compute the center of curvature $\mathbf{x}_{\kappa}$. Initial parameters for the fitting process are determined from the estimated parameters of the previous segment using a Kalman filter, i.e., the incremental scheme adjusts for varying thickness, changing direction, and changing curvature.

\section{Experimental Results}

We have applied our new approach to 3D synthetic images as well as 3D CTA image data. For the synthetic experiments, we have used 3D images containing different curved tubular structures such as tori and spirals with a range of different parameter settings. For example, we have generated 3D spirals using a cross-section radius of $R=1$, a contrast of $a=100$, a standard deviation of the Gaussian smoothing of $\sigma=1$, and different levels of additive Gaussian noise with standard deviations of $\sigma_{n}=0,1,3,5,10$. Note that a spiral has a varying curvature along the tubular structure. For example, Fig. 1(a) shows the segmentation result for a $3 \mathrm{D}$ spiral. In addition, Fig. 1 displays a $2 \mathrm{D}$ section of the segmentation results highlighting the inner part of the spiral $\left(\sigma_{n}=5\right)$ using (b) a previous straight model and (c) our new curved model. Shown are the centerline 
Table 1. Mean error $\bar{e}$ and maximal error $e_{\max }$ of the estimated centerline position $\mathbf{x}_{0}$, the radius $R=\sigma$, and the radius of curvature $R_{\kappa}$ for five different noise levels $\left(\sigma_{n}=0,1,3,5,10\right)$ for a previous straight model (left) and the new model (right).

\begin{tabular}{llllllllllll}
\hline \multicolumn{4}{c}{ Straight tubular model } & \multicolumn{8}{c}{ Curved tubular model } \\
$\sigma_{n}$ & $\bar{e}_{\mathbf{x}_{0}}$ & $e_{\max , \mathbf{x}_{0}}$ & $\bar{e}_{R}$ & $e_{\max , \mathrm{R}}$ & $\bar{e}_{\mathbf{x}_{0}}$ & $e_{\max , \mathbf{x}_{0}}$ & $\bar{e}_{R}$ & $e_{\max , \mathrm{R}}$ & $\bar{e}_{\kappa}$ & $e_{\max , \kappa}$ \\
\hline 0 & 0.21 & 0.92 & 0.14 & 0.32 & 0.01 & 0.04 & 0.13 & 0.13 & 0.0005 & 0.0159 \\
1 & 0.21 & 0.92 & 0.14 & 0.32 & 0.01 & 0.05 & 0.13 & 0.14 & 0.0010 & 0.0155 \\
3 & 0.21 & 0.93 & 0.14 & 0.32 & 0.04 & 0.11 & 0.13 & 0.16 & 0.0029 & 0.0146 \\
5 & 0.21 & 0.93 & 0.14 & 0.33 & 0.06 & 0.19 & 0.13 & 0.19 & 0.0047 & 0.0187 \\
10 & 0.22 & 0.94 & 0.14 & 0.35 & 0.12 & 0.41 & 0.13 & 0.26 & 0.0095 & 0.0390 \\
\hline
\end{tabular}

(black) and the inner and outer contours (white). The spiral has been quite well segmented using the curved model. In contrast, the straight model yields a worse result, i.e., the estimated centerline and contours are shifted inwards with increasing curvature.

To quantify the segmentation results, we have computed the mean error $\bar{e}$ and the maximal error $e_{\max }$ of the estimated centerline position $\mathbf{x}_{0}$, the radius $R=\sigma$, and the curvature $\kappa$ along the spiral. Table 1 shows the results for five different noise levels $\left(\sigma_{n}=0,1,3,5,10\right)$. From the experiments we found that our curved tubular model generally yields accurate results for the centerline position, radius, and curvature. The maximal errors of the centerline position $\left(e_{\max , \mathbf{x}_{0}}=0.41\right.$ voxels $)$ and of the radius $\left(e_{\max , \mathrm{R}}=0.26\right.$ voxels $)$ for a relatively high level of noise $\left(\sigma_{n}=10\right)$ are well in the subvoxel range, and even lower for less image noise. Also the error of the curvature is quite small, i.e., the maximal error is only about $10 \%$ of the true value. In comparison, for the straight model significantly larger maximal errors of the position $\left(e_{\max , \mathbf{x}_{0}}=0.94\right.$ voxels $)$ and of the radius ( $e_{\max , \mathrm{R}}=0.35$ voxels) are obtained. Moreover, Fig. 1(d) shows the error $e_{\mathbf{x}_{0}}$ of the centerline position along the spiral for the previous straight model (grey) and our new curved model (black). For the previous model, the error increases strongly along the spiral, whereas for the new model the error remains relatively small.

We have also applied the new approach to different 3D CTA vascular images of the human. Figs. 2a,b show the segmentation result for a small vessel in a 3D CTA image of a human brain. For two positions on the centerline the estimated curvature is visualized by displaying the osculating circle as a torus (bright ring). The tori are defined by the estimated radius of curvature $R_{\kappa}$, center of curvature $\mathbf{x}_{\kappa}$, and radius $R=\sigma$. In the figure, examples are shown for a high curvature (a) and a low curvature (b) vessel segment. In addition, the segmentation result of a curved small vessel feeding a liver tumor is presented in Fig. 2(c). It turned out that vessels of varying curvatures have been well segmented. 
Fig. 2. Segmentation results for a small vessel in a 3D CTA of a human brain where osculating circles (bright rings) highlight the local curvature for a vessel segment of (a) high and (b) low curvature as well as for (c) a curved small vessel feeding a tumor.

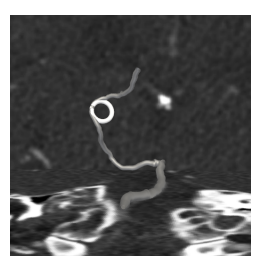

(a)

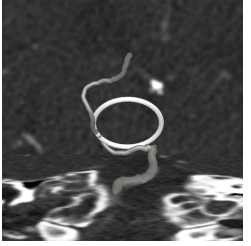

(b)

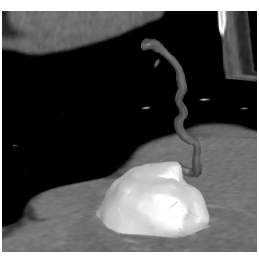

(c)

\section{Discussion}

We introduced a new curved tubular intensity model in conjunction with a model fitting scheme for accurate segmentation and quantification of small tubular structures in 3D vascular images. The tubular model includes a parameter for the local (extrinsic) curvature of tubular structures. We have proposed to formulate the curved tubular model as a 3D Gaussian smoothed Dirac ring, and we have derived an analytic solution for the model function. In contrast to previous straight models, the new model allows to represent curved tubular structures, to directly estimate the local curvature by model fitting, as well as to more accurately estimate the remaining model parameters such as the radius and centerline of tubular structures. We have successfully applied our approach to $3 \mathrm{D}$ synthetic images as well as 3D CTA images of the human vascular system.

Acknowledgement. This work has been funded by the Deutsche Forschungsgemeinschaft (DFG) within the project QuantVessel (RO 2471/6).

\section{References}

1. Sato Y, Nakajima S, Shiraga N, et al. Three-dimensional multi-scale line filter for segmentation and visualization of curvilinear structures in medical images. Med Image Anal. 1998;2(2):143-168.

2. Manniesing R, Velthuis BK, van Leeuwen MS, et al. Level set based cerebral vasculature segmentation and diameter quantification in CT angiography. Med Image Anal. 2006;10:200-214.

3. Frangi AF, Niessen WJ, Hoogeveen RM, et al. Model-based quantitation of 3D magnetic resonance angiographic images. IEEE Trans Med Imaging. 1999;18(10):946956.

4. Kang DG, Suh DC, Ra JB. Three-dimensional blood vessel quantification via centerline deformation. IEEE Trans Med Imaging. 2009;28(3):405-414.

5. Noordmans HJ, Smeulders AWM. High accuracy tracking of 2D/3D curved line structures by consecutive cross-section matching. Pattern Recognit Lett. 1998;19(1):97-111.

6. Wörz S, Rohr K. Segmentation and quantification of human vessels using a 3-D cylindrical intensity model. IEEE Trans Image Process. 2007;16(8):1994-2004. 